\title{
Brown dwarf disks with ALMA: evidence for truncated dust disks in Ophiuchus (Corrigendum)
}

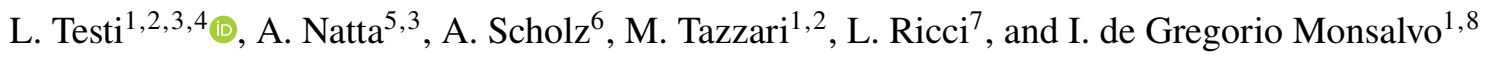 \\ ${ }^{1}$ ESO/European Southern Observatory, Karl-Schwarzschild-Strasse 2, 85748 Garching bei München, Germany \\ e-mail: 1testi@eso.org \\ 2 Excellence Cluster "Universe", Boltzmann str. 2, 85748 Garching bei Muenchen, Germany \\ ${ }^{3}$ INAF/Osservatorio Astrofisico of Arcetri, Largo E. Fermi, 5, 50125 Firenze, Italy \\ ${ }^{4}$ Gothenburg Center for Advance Studies in Science and Technology, Department of Mathematical Sciences, Chalmers University of \\ Technology and University of Gothenburg, 41296 Gothenburg, Sweden \\ ${ }^{5}$ School of Cosmic Physics, Dublin Institute for Advanced Studies, 31 Fitzwilliams Place, Dublin 2, Ireland \\ ${ }^{6}$ SUPA, School of Physics and Astronomy, University of St. Andrews, North Haugh, St. Andrews, Fife KY16 9SS, UK \\ ${ }^{7}$ Harvard-Smithsonian Center for Astrophysics, 60 Garden Street, Cambridge, MA 02138, USA \\ ${ }^{8}$ Joint ALMA Observatory (JAO), Alonso de Cordova 3107 Vitacura -Santiago de Chile, Chile
}

A\&A, 593, A111 (2016), https://doi .org/10.1051/0004-6361/201628623

Key words. protoplanetary disks - brown dwarfs - stars: formation - submillimeter: planetary systems - errata, addenda

An error occurred in the paper: the object ISO-Oph 164 was not observed by ALMA as part of project 2012.1.00037.S. The object observed (see the ALMA image in Fig. 1) was instead GY92 317 (Greene \& Young 1992). This object is probably a field star, as discussed in Barsony et al. (1997). The upper limit for the millimetre flux of ISO-Oph 164 reported in Table 2 is thus incorrect.
None of the conclusions of the paper is affected by this error.

\section{References}

Barsony, M., Kenyon, S. J., Lada, E. A., \& Teuben, P. J. 1997, ApJS, 112, 109 Greene, T. P., \& Young, E. T. 1992, ApJ, 395, 516 